\title{
Scaling theory and computer simulation of star polymers in good solvents
}

\author{
K.Ohno \\ Department of Physics, Faculty of Engineering, \\ Yokohama National University, \\ 79-5 Tokiwadai, Hodogaya-ku, Yokohama 240-8501, Japan
}

Received December 7, 2001

The scaling theories and the results of the renormalization-group $\varepsilon=4-d$ expansion ( $d$ is the spatial dimensionality) as well as the computer simulations such as Monte Carlo simulations are extensively reviewed for star polymers with very long flexible arms of equal length in a dilute solution of the good solvent limit, with a close connection to general polymer networks. In particular, the asymptotic behaviour of the conformational and entropic quantities in the long chain limit is discussed in detail in terms of the polymer-magnetism analogy. Discussions are given not only for static properties such as the distribution functions and the osmotic pressure or entropy but also for dynamic properties such as the relaxation time and the intrinsic viscosity of star polymers.

Key words: renormalization group, Monte Carlo simulation, total number of configurations, virial coefficient, relaxation time, hydrodynamic effect

PACS: $05.10 . C c, 05.10 . L n, 61.41 .+e, 82.35 . G h, 82.35 . L r, 82.70 . U v$

\section{Introduction}

The complicated statistics of polymer networks [1] having ring [2], star [3], and other branched topologies in solution have received a continuous attention over a long time. The statistics of polymer networks resolved in a good solvent in the dilute limit can be measured in terms of monomer distribution, end-to-end distance distribution, entropy, and so on. On the other hand, typical dynamic properties of a dilute solution of polymer networks in a good solvent are as follows: relaxation time, diffusion constant, viscosity, sedimentation coefficient, hydrodynamic radius, and so on. They can be measured experimentally by means of optical, neutron-diffraction and other techniques. They are theoretically estimated by means of computer simulations and other numerical techniques (see [4,5] for review and see also [6-14] for star polymers) as well as using sophisticated analyses such as the exact results in two dimensions 
$[15,16]$, scaling theory $[2,17-19]$ and renormalization-group $(\mathrm{RG})$ techniques (for star polymers see [20-25] as well as [17,19], and for other polymer networks see $[15,26])$. All these quantities and techniques are of course not special for polymer networks but have been rather common for linear polymers. However, the resulting behaviour is not always similar to that of linear polymers. As an example, a ring polymer having a very simple polymer architecture, which is characterized by a closed loop without branch, is known to have the statistics in a dilute solution different from that of an open chain [2]. Another extreme example of polymer networks is a polymer gel, which is a huge network of many flexible linear chains, and its statistics and dynamics have attracted interest [2].

A star polymer is composed of many long arm chains starting from a center unit. Still it has a simple enough structure but its statistics and dynamics involve a lot of important ingredients of more general polymer networks. In fact, it has been found in two $[15,16]$ and arbitrary dimensions $[15,17,18]$ that the behaviour of the total number of configurations of star polymers determines the behaviour of general polymer networks, The study of star polymers is also interesting in other respects since it has a close relationship to the subject of micellar and other polymeric surfactant systems [27-30], and has a potential to bring widespread applications.

In the past two decades, the great progress in the synthesis of highly qualified monodisperse polymer networks [31-38] has stimulated many experimental and theoretical studies of star polymers. More recently, star polymers with a huge number of arms has also been synthesized in a controlled fashion [39]. The experimental studies of star polymers have stimulated theoretical studies to be more closely tied up with the experiments. For example, the virial coefficients [40-42], relaxation times $[43,44]$ and hydrodynamic effects [45-48] have been more recently investigated with a powerful tool of computer simulations and some of their results have been successfully compared with the experiments $[31-37,49,50]$ (for a recent review, see [51]). The effect of walls or other confining geometries has also found interest [52-54] in connection, for example, with surface critical phenomena (see [55] for a review). The center-absorbed star polymers are also related to the polymer brush [56] when the interchain distances on the substrate become short enough. In this article, the study of star polymers in good solvents in a dilute regime will be reviewed from the theoretical standpoint.

\section{Lattice model and magnetic analogy of polymer networks}

The theory of linear polymers in a good solvent has been very successful as a result of the connection established by de Gennes [2] and des Cloizeaux [57] between the polymer statistics and the critical phenomena of an $n$-component classical spin model in the $n \rightarrow 0$ limit. The discussions presented in this section can be seen mostly in [17-19]. 


\subsection{Lattice chain model and $n$-vector model}

Let us first start from a lattice chain model and assume that there are $f$ chains of length $l_{1}, l_{2}, \ldots, l_{f}$ on a regular lattice. To be specific, let us use a $d$-dimensional hypercubic lattice with a unit lattice constant ( $d=3$ for usual purposes). Each segment has a unit length and can rotate only by $90^{\circ}$. If the position of a joint of segments at a contour distance $s_{i}$ along the $i$ th lattice chain is designated by $\boldsymbol{r}\left(s_{i}\right)$, the Hamiltonian of this lattice chain system can be written as

$$
H=a \sum_{i=1}^{f} \sum_{s_{i}=1}^{l_{i}-1}\left\{\left[\boldsymbol{r}\left(s_{i}+1\right)-\boldsymbol{r}\left(s_{i}\right)\right]^{2}-1\right\}+b \sum_{i=1}^{f} \sum_{j=1}^{f} \sum_{s_{i}=1}^{l_{i}-1} \sum_{s_{j}=1}^{l_{j}-1} \delta \boldsymbol{r}\left(s_{i}\right) \boldsymbol{r}\left(s_{j}\right),
$$

where $\delta_{\boldsymbol{r}}\left(s_{i}\right) \boldsymbol{r}\left(s_{j}\right)$ means Kronecker's delta and the parameters $a$ and $b$ are chosen to be $0<a<b$. Then, we see that the energy is $b-a(>0)$, zero, $a(>0), 2 a(>0), 3 a(>0)$, $\ldots$, respectively, when the distance between two adjacent joint points is $0,1, \sqrt{2}$, $\sqrt{3}, 2$ and so on. And, if two joint points, which are on two different chains or on the same chain but apart from each other along the chain contour, meet at the same point, the energy is increased by $b(>0)$. Therefore, keeping the conformations with zero energy only, we obtain $f$ chains which do not pass the same lattice point twice, i.e., satisfying the self-avoiding condition. They are called self-avoiding walks (SAWs) on a lattice. We want to count the number of all such configurations with a given set of fixed chain-end points, $O_{i}=\boldsymbol{r}\left(s_{i}=1\right)$ and $P_{i}=\boldsymbol{r}\left(s_{i}=l_{i}\right), i=1,2, \ldots, f$. This is possible when we estimate the "restricted" partition function

$$
\mathcal{N}\left(O_{1} P_{1}, O_{2} P_{2}, \ldots, O_{f} P_{f} ; l_{1}, l_{2}, \ldots, l_{f}\right)=\lim _{T \rightarrow 0} \prod_{i=1}^{f} \prod_{s_{i}=2}^{l_{i}-1} \sum_{\left\{\boldsymbol{r}\left(s_{i}\right)\right\}} \exp \left[-\frac{H}{k_{\mathrm{B}} T}\right]
$$

of this Hamiltonian at $T=0$. (The meaning of "restricted" is that the sum with respect to joint points $\left\{\boldsymbol{r}\left(s_{i}\right)\right\}$ over the lattice points is taken with fixed chain ends at $O_{i}=\boldsymbol{r}\left(s_{i}=1\right)$ and $P_{i}=\boldsymbol{r}\left(s_{i}=l_{i}\right), i=1,2, \ldots, f$.) That is, the total number of configurations of $f$-chains on a lattice with fixed end points is exactly given by equation (2) at $T=0$. From the complete knowledge on the total number of configurations, one can calculate various averages of geometrical quantities of polymer networks.

Physical polymer networks contain loops and branch points, while the $f$ linear chains introduced above are disconnected. However, by requiring some of the end points of linear chains to be in close proximity of each other, one can obtain desired structures of the considered network. If $O_{1}, O_{2}, \ldots, O_{f}$ are put as the nearest neighbours of each other while the positions of $P_{1}, P_{2}, \ldots, P_{f}$ are not restricted, an $f$-arm star geometry is realized. In fact, such a proximity constraint has the same physical effect as putting in suitable chemical crosslinks (functional units) to form the network. In what follows, we will consider a general polymer topology $\mathcal{G}$ and treat each crosslink, at which several chains meet at close vicinities, as a single branch point as a whole. 
Total number of configurations of a polymer network with a fixed topology $\mathcal{G}$ is

$$
\mathcal{N}_{\mathcal{G}}\left(l_{1}, l_{2}, \ldots, l_{f}\right)=\frac{1}{N} \sum_{\text {end and branch points }} \mathcal{N}\left(O_{1} P_{1}, O_{2} P_{2}, \ldots, O_{f} P_{f} ; l_{1}, l_{2}, \ldots, l_{f}\right),
$$

where the summation with respect to end and branch points is taken over all $N$ lattice points. The prefactor $1 / N$ is necessary because there are $N$ translationally identical configurations which should not be distinguished. It is often convenient to introduce a slightly different definition for the total number of configurations in which the total length of chains is fixed at $L$ :

$$
\mathcal{N}_{\mathcal{G}}(L)=\sum_{l_{1}=0}^{\infty} \sum_{l_{2}=0}^{\infty} \cdots \sum_{l_{f}=0}^{\infty} \mathcal{N}_{\mathcal{G}}\left(l_{1}, l_{2}, \ldots, l_{f}\right) \delta_{l_{1}+l_{2}+\ldots+l_{f}, L}
$$

Now that the lattice chain model is defined completely, one can demonstrate its relation to the lattice spin model called the $n$-vector model. To this end, we define the generating function for the total number of configurations as

$$
Z_{\mathcal{G}}(K)=\sum_{L=0}^{\infty} \mathcal{N}_{\mathcal{G}}(L) K^{L}
$$

This generating function (5) is proved to be equal exactly to a nonlinear susceptibility (in the limit $n \rightarrow 0$ ) of the $n$-vector model:

$$
Z_{\mathcal{G}}(K)=\chi_{\mathcal{G}}(K), \quad(n \rightarrow 0)
$$

The $n$-vector model is defined by the Hamiltonian

$$
\begin{aligned}
\mathcal{H} & =-J H=-k_{\mathrm{B}} T K H, \quad K=\frac{J}{k_{\mathrm{B}}}, \\
H & =\sum_{\langle i j\rangle} \boldsymbol{S}_{i} \cdot \boldsymbol{S}_{j} .
\end{aligned}
$$

In equation (8), the summation runs over all the nearest-neighbour pairs, $i j$, on a lattice, and each $\operatorname{spin} \boldsymbol{S}_{i}$ at each lattice point $i$ has $n$ components

$$
\boldsymbol{S}_{i}=\left(S_{i}^{(1)}, S_{i}^{(2)}, \ldots, S_{i}^{(n)}\right)
$$

and fixed length $\left|\boldsymbol{S}_{i}\right|^{2}=n$. This Hamiltonian describes the Ising model, the planar (XY) model and the classical Heisenberg model, respectively, for $n=1,2$ and 3 .

The relevant nonlinear susceptibility $\chi_{\mathcal{G}}(K)$ can be constructed as follows: consider the system of $f$ linear chains which do not have contacts with each other and suppose that the polymer network has $n_{k}, k$-functional units. The $k$-functional unit around point $P$ consists of $g$ neighbouring points $P_{1}, P_{2}, \ldots, P_{k}$, at which each linear chain starts. Then we are requested to introduce the $k$ th order composite operator

$$
\Psi_{k}^{\left(m_{1}, m_{2}, \ldots, m_{k}\right)}=\sum_{P} S_{P_{1}}^{\left(m_{1}\right)} S_{P_{2}}^{\left(m_{2}\right)} \ldots S_{P_{k}}^{\left(m_{k}\right)}
$$


for each $g$-functional unit constituting the network with the topology $G$. The sum with respect to $P$ is performed with a fixed vertex structure, because the neighbouring points $P_{1}, P_{2}, \ldots, P_{k}$ do not change their configuration around $P$. Then, for a given topology $G$ of polymer networks, the nonlinear susceptibility is given by

$$
\chi_{\mathcal{G}}(K)=\frac{1}{N}\left\langle\prod_{k}\left[\Psi_{k}^{\left(m_{1}, m_{2}, \ldots, m_{k}\right)} \Psi_{k}^{\left(m_{1^{\prime}}, m_{2^{\prime}}, \ldots, m_{k^{\prime}}\right)} \ldots \Psi_{k}^{\left(m_{1^{\prime \prime}}, m_{2^{\prime \prime}}, \ldots, m_{k^{\prime \prime}}\right)}\right]\right\rangle .
$$

One should recall that $i$ th chain carries $i$ th spin component, so that the same component appears just twice in the brackets on superscript of $\Psi$ 's in (10).

When the nonlinear susceptibility $\chi_{\mathcal{G}}(K)$ is calculated with the renormalization group ( $\mathrm{RG}$ ) theory, the average of the product of composite operators becomes, after renormalization, a linear combination of the original function and other functions which are related to simpler topologies obtained from the original topology $\mathcal{G}$ by shrinking some arms and have the same or lower canonical dimensions. If we are interested in the networks having the same chain length $l$, we should discard all these terms which occur due to additive renormalization. That is, it is only necessary to identify the renormalization factor associated with the topology $\mathcal{G}$ itself. We will call this part of the renormalized nonlinear susceptibility the essential part.

\subsection{Relation to critical phenomena}

The equivalence between polymer and magnetic systems enables us to study conformational and entropic properties of polymer networks of long flexible chains.

From the knowledge of the $n$-vector model, we expect that the nonlinear susceptibility (10) generally exhibits a singular behaviour like

$$
\chi_{\mathcal{G}}(K) \sim t^{\hat{\gamma}_{\mathcal{G}}}
$$

Here $t$ is the reduced temperature defined by $\mu K=\mathrm{e}^{-t}$ with $\mu=1 / K_{\mathrm{c}}=k_{\mathrm{B}} T / J$; $\mu$ is a parameter proportional to the critical temperature of the $n$-vector model in the limit $n \rightarrow 0$. Note that $t=0$ corresponds to the critical point $K=K_{\mathrm{c}}$ and $t \sim 1-\mu K$ holds only near the critical point.

If we replace the summation with respect to $L$ in (5) by an integration and use $\mu K=\mathrm{e}^{-t}$, the relation (6) becomes a form of Laplace transformations: $\chi_{\mathcal{G}}(K) \sim$ $\int_{0}^{\infty} \mathrm{d} L\left[\mathcal{N}_{\mathcal{G}}(L) / \mu^{L}\right] \mathrm{e}^{-t L}$, where a symbol $\sim$ is used to indicate its validity only in the scaling limit, $L \rightarrow \infty$. Because the nonlinear susceptibility is expected to have a power-law singularity (11) near $t \sim 0$, an inverse-Laplace-transformation gives

$$
\mathcal{N}_{\mathcal{G}}(L) \sim L^{\hat{\gamma}_{\mathcal{G}}-1} \mu^{L}
$$

Thus, the exponent $\hat{\gamma}_{\mathcal{G}}$ for the total number of configurations of networks with a fixed total length $L$ is found to be identical to the exponent of the nonlinear susceptibility (11). In enumerations of SAWs on a lattice, the constant $\mu$ is sometimes called the effective coordination number. Note that, in the case of simple random walks, $\mu$ is equal to the coordination number of a lattice ( $2 d$ for the $d$-dimensional 
hypercubic lattice) and $\hat{\gamma}_{\mathcal{G}}$ is equal to 1 . In contrast, in the case of SAWs, $\mu$ is slightly smaller than the coordination number minus 1 , the number of possible directions of elongating a chain end by one segment without folding backward onto itself. This mapping shows that $\mu$ is independent of the topology of the polymer network.

As a more important case, when the length of every single chain is all the same and given by $l$, the total number of configurations $\mathcal{N}_{\mathcal{G}}(l, l, \ldots, l)$ behaves differently from the $\mathcal{N}_{\mathcal{G}}(L)$ with a fixed total chain length $L$. The behaviour of this $\mathcal{N}_{\mathcal{G}}(l, \ldots, l)$ may be explored by recognizing that, if all chain lengths $l_{1}, \ldots, l_{f}$ are of the same order, then $\mathcal{N}_{\mathcal{G}}\left(l_{1}, \ldots, l_{f}\right)$ behaves like $\left(l_{1}+\cdots+l_{f}\right)^{\hat{\gamma}_{\mathcal{G}}-1} \mu^{l_{1}+\cdots+l_{f}}$. Then, since such a region dominates in the sum of $(4)$, we obtain

$$
\mathcal{N}_{\mathcal{G}}(L) \sim L^{\gamma_{\mathcal{G}}-1} \mu^{L} \sum_{l_{1}=0}^{\infty} \sum_{l_{2}=0}^{\infty} \cdots \sum_{l_{f}=0}^{\infty} \delta_{l_{1}+l_{2}+\cdots+l_{f}, L} \sim L^{\gamma_{\mathcal{G}}+f-2} \mu^{L} .
$$

The comparison between (12) and (13) yields

$$
\gamma_{\mathcal{G}}=\hat{\gamma}_{\mathcal{G}}+1-f
$$

Let us turn our attention to the end-to-end distribution of a general polymer network $\mathcal{G}$. We consider the total number of configurations $\mathcal{N}_{\mathcal{G}}\left(O_{i}, P_{j} ; L\right)$ which is defined by the $\mathcal{N}_{\mathcal{G}}(L)$ summed up with respect to all ends and branch points except $O_{i}$ and $P_{j}$. It depends on spatial coordinates only through the distance $r_{i j}$ between $O_{i}$ and $P_{j}$. Then, introducing the reduced temperature and the Laplace transformation, and applying the magnetic analogy for this function, we are led to the local nonlinear susceptibility $\chi_{\mathcal{G}}\left(O_{i}, P_{j} ; K\right)$ expressed as $\sim \int_{0}^{\infty} \mathrm{d} L\left[\mathcal{N}_{\mathcal{G}}\left(O_{i}, P_{j} ; L\right) / \mu^{L}\right] \mathrm{e}^{-t L}$. From the scaling theory of spin systems, we expect that it obeys a scaling form

$$
\chi_{\mathcal{G}}\left(O_{i}, P_{j} ; K\right) \sim \frac{1}{r_{i j}^{d-2+\eta_{\mathcal{G}}}} \varphi_{\mathcal{G}}\left(\frac{r_{i j}}{t^{-\nu}}\right),
$$

where $\nu$ is the correlation-length exponent; and $\eta_{\mathcal{G}}$ is the anomalous dimension and is related to $\hat{\gamma}_{\mathcal{G}}$ via $\hat{\gamma}_{\mathcal{G}}=\nu\left(2-\eta_{\mathcal{G}}\right)$. Then, an inverse-Laplace-transformation gives $\mathcal{N}_{\mathcal{G}}\left(O_{i}, P_{j} ; L\right) \sim\left(\mu^{L} / L r_{i j}^{d-2+\eta_{\mathcal{G}}}\right) \tilde{\varphi}_{\mathcal{G}}\left(r_{i j} L^{-L}\right)$. Finally, dividing it by $\mathcal{N}_{\mathcal{G}}(L)$, we find the end-to-end distribution function for fixed topology $\mathcal{G}$ and fixed total length $L$,

$$
p_{\mathcal{G}}\left(r_{i j}\right)=\frac{\mathcal{N}_{\mathcal{G}}\left(O_{i}, P_{j} ; L\right)}{\mathcal{N}_{\mathcal{G}}(L)} \sim \frac{1}{L^{\hat{\gamma}} r_{i j}^{d-2+\eta_{\mathcal{G}}}} \hat{\phi}_{\mathcal{G}}\left(r_{i j} L^{-L}\right)=\frac{1}{r_{i j}^{d}} \phi_{\mathcal{G}}\left(r_{i j} L^{-\nu}\right),
$$

similar to the single chain problems. The distribution function for general polymer networks where all chains have the same length $l$ is also expected to have the same form as (16). From (16), we get to the mean square end-to-end distance

$$
\left\langle r_{i j}^{2}\right\rangle \sim L^{2 \nu},
$$

which also behaves like a single polymer chain. Thus, we expect that if a flexible polymer network made of connecting very long chains is desolved in a good solvent, its gyration radius is characterized by the same exponent $\nu$ as for a single selfavoiding walk (SAW) (0.588 for $d=3$ ) irrespective of the network structure $[2,17]$. 


\subsection{Scaling theory of general polymer networks}

Although the gyration radius exponent $\nu$ of an arbitrary polymer network is the same as that of a single self-avoiding walk (SAW), the exponent for the total number of configuration is generally different from that of the SAW and depends on the topology of the network $\mathcal{G}$.

To see this, we will discuss a phenomenological scaling theory of general polymer networks. We assume that the $g$ th-order composite field $h_{g}$, which is conjugate to the $g$ th-order composite operator $\Psi_{g}$, scales as $t^{\Delta_{g}}$ irrespective of their components. That is, we assume that the free energy of the system has a scaling form

$$
F \sim t^{2-\alpha} \Phi\left(\frac{h_{1}}{t^{\Delta_{1}}}, \frac{h_{2}}{t^{\Delta_{2}}}, \ldots\right)
$$

where $\alpha$ is the specific heat exponent of the spin system $(\alpha \sim 0.23$ for $d=3)$ and is related to $\nu$ by the hyperscaling relation $\alpha=2-\nu d$

The nonlinear susceptibility with composite operators is generally not multiplicatively renormalizable but mixed with other functions which have the same or lower canonical dimension [58]. Correspondingly, in the configurations of the network $\mathcal{G}$ with a fixed total length $L$, many simpler topologies are realized by shrinking some of the linear chains in $\mathcal{G}$. In order to discuss the configurations with all chain lengths being the same, one should discard all such simpler (and less singular) terms and preserve only the singularity associated with the topology $\mathcal{G}$ (the essential part). The corresponding exponent $\gamma_{\mathcal{G}}$ is obtained from the exponent $\hat{\gamma}_{\mathcal{G}}$ of the essential part associated with the topology $\mathcal{G}$ of the nonlinear susceptibility via equation (14).

We have the essential part of the nonlinear susceptibility behaving as

$$
\chi_{\mathcal{G}}(K) \sim-\left.\frac{1}{N}\left[\prod_{g} \frac{\partial^{n_{g}}}{\partial h_{g}^{n_{g}}}\right] F\right|_{h=0} \sim t^{-\hat{\gamma}_{\mathcal{G}}}
$$

with $\hat{\gamma}_{\mathcal{G}}=\alpha-2+\sum_{g} n_{g} \Delta_{g}$. Then using the relation (14), the exponent $\gamma_{\mathcal{G}}$ for the total number of configurations of $f$ chains having the same length $l$ is given by

$$
\gamma_{\mathcal{G}}=\alpha-1-f+\sum_{g=1}^{\infty} n_{g} \Delta_{g}
$$

This expression was first obtained by Duplantier [15] who used the two-dimensional exact analysis and the renormalization-group $(\mathrm{RG})$ approach. The present derivation is based on a phenomenological scaling argument given by Ohno and Binder [17].

If we consider a comb polymer composed of $g=(f-1) / 23$-functional units and $g+2$ side branches, its exponent is given by $\gamma_{\mathrm{comb}}(g)=\gamma+g[\gamma(3)-\gamma]$ from the scaling relations (20) and (21). This expression is verified to $\mathcal{O}(\varepsilon)$ in the $\mathrm{RG} \varepsilon=4-d$ expansion, if we use $\gamma(3)$, which was calculated specifically for star polymers to $\mathcal{O}(\varepsilon)$ by Miyake and Freed $\left[20,21\right.$ ] [see also $(22)$ in the next section], and $g_{\text {comb }}(g)$, which was calculated specifically for comb polymers to $\mathcal{O}(\varepsilon)$ by Vlahos and Kosmas [26]. 


\subsection{Scaling behaviour of star polymers}

Let us discuss the scaling behaviour of star polymers. First of all, the exponent $\gamma_{\mathcal{G}} \equiv \gamma(f)$ for an $f$-arm star polymer is expressed by the scaling relation (20) as

$$
\gamma(f)=\alpha-1+\frac{f}{2}(\gamma-\alpha)+\Delta_{f}
$$

Thus, combining (20) and (21), one can express $\gamma_{\mathcal{G}}$ for an arbitrary polymer network $\mathcal{G}$ in terms of well known exponents $\gamma, \nu$ and $\alpha$, and star polymer exponents $\gamma(f)$.

Now we mention the result of the RG $\varepsilon$ expansion for the star polymer exponents. For the exponent $\gamma(f)$, the first-order term in $\varepsilon$ was determined in 1983 by a pioneering work of Miyake and Freed [20,21]. The expression valid up to the second order in $\varepsilon$ was obtained by Ohno and Binder [17] as follows:

$$
\begin{aligned}
& \gamma(f)=1+(\gamma-1)\left[f-\frac{f(f-1)}{2}\right]+f(f-1)(f-2) A(f), \\
& A(f)=\frac{1}{64} \varepsilon^{2}+\mathcal{O}\left(\varepsilon^{3}\right)
\end{aligned}
$$

$A(f)$ is a regular function of $f$. Its value at $\mathcal{O}\left(\varepsilon^{3}\right)$ was identified by Duplantier [24]. The expansion is, however, an asymptotic expansion and does not converge even at small $\varepsilon$ and $f$ [25]. The large $f$ behaviour of $\gamma(f)$ was predicted by Ohno [23] to be $\gamma(f) \sim-f^{d /(d-1)}$ for arbitrary dimensions, $2 \leqslant d<4$. The $\gamma(f)$ was also evaluated by Monte Carlo techniques [7,11,13] (see section 3.1).

It is possible to discuss additional scaling relations which relate the contact exponents of a linear chain to the star-polymer exponents. Using the scaling relations (20) and (21), Duplantier and Saleur [59] and Ohno and Binder [17] obtained

$$
\gamma(3)=2 \gamma-1-\nu \theta_{1}, \quad \gamma(4)=2 \gamma-1-\nu \theta_{2},
$$

where $\theta_{1}$ denotes the contact exponent characterizing the short distance behaviour (s.d.b.) between one end and one interior point of a linear chain; and $\theta_{2}$ characterizes the s.d.b. between two interior points of a linear chain. The scaling relations (24) between contact and star-polymer exponents are fulfilled if we use (22) with (23) for $\gamma(f)$ and the contact exponents calculated up to $\mathcal{O}\left(\varepsilon^{2}\right)$ by des Cloizeaux [60].

For a star polymer, the mean distance $R_{\mathrm{C}}$ of an arbitrary monomer $j$ from the center, the radius of gyration of the total polymer $R_{\text {gyr }}$, and the mean center-end distance $R_{\mathrm{CE}}$ are predicted by Daoud and Cotton [3] to behave as [see also (17)]

$$
R_{\mathrm{C}} \sim R_{\mathrm{gyr}} \sim R_{\mathrm{CE}} \sim f^{\sigma} l^{\nu}, \quad \sigma=\frac{1-\nu}{d-1} .
$$

The short distance behaviours of the monomer density distribution function

$$
\rho(\mathbf{r})=\frac{1}{r^{d-1 / \nu}} \psi\left(\frac{r}{R_{\mathrm{C}}}\right),
$$


and the center-end distribution function

$$
g\left(\mathbf{r}^{\mathrm{CE}}\right)=\frac{1}{R_{\mathrm{CE}}^{d}} \phi\left(\frac{r^{\mathrm{CE}}}{R_{\mathrm{CE}}}\right),
$$

which are normalized as $\int \mathrm{d} \mathbf{r}^{\mathrm{CE}} g\left(\mathbf{r}^{\mathrm{CE}}\right)=1$ and $\int \mathrm{d} \mathbf{r} \rho(r)=L$, are given by [18]

$$
\begin{aligned}
& \psi(x)=\mathrm{const}, \quad \phi(x) \sim x^{\theta(f)}, \quad(\text { for } x \ll 1) \\
& \theta(f)=[\gamma-\gamma(f+1)+\gamma(f)-1] / \nu ;
\end{aligned}
$$

$\gamma$ being the configuration number exponent of a free linear polymer. Equations (26)(29) refer to free star polymers only; a more general form is discussed in [61].

\subsection{Star polymers and polymer networks in semi-infinite geometry}

The scaling theory for general polymer networks can be generalized to the case where some end or branch points are grafted on a flat surface [16-19]. In the last two sections, we saw that the exponent $\gamma_{\mathcal{G}}$ for a complicated network can be expressed as a linear combination of only these star polymer exponents $\gamma(f)$, and as the wellknown exponents of single linear polymer chains. Similarly, the exponent $\gamma_{\mathcal{G}}$ for a polymer network $\mathcal{G}$ which has $n_{h} h$-functional units free, $n_{h}^{\prime} h$-functional units grafted at the surface and totally $f$ linear polymers with the same length obeys the scaling relation

$$
\gamma_{\mathcal{G}}=\alpha-1-f+\nu+\sum_{h=1}^{\infty}\left[n_{h} \Delta_{h}+n_{h}^{\prime} \Delta_{h}^{\prime}\right] .
$$

This scaling relation was first obtained for two dimensions by Duplantier and Saleur [16] and then for arbitrary dimensions by Ohno and Binder [17].

For center-absorbed stars $\gamma_{\mathbf{s}}(f)$ is used for $\gamma_{\mathcal{G}}$, and for stars which have one, two, ... ends of arms grafted at the wall, $\gamma_{1}(f), \gamma_{11}(f)$, etc., are used. From $(30), \gamma_{\mathbf{s}}(f)$, $\gamma_{1}(f), g l l(f), \ldots$ are expressed, by assuming that $g_{11 \ldots 1}(f)$ has $g$ subscripts 1 , as

$$
\begin{aligned}
& \gamma_{\mathrm{s}}(f)=\alpha-1+\nu+\frac{f}{2}(\gamma-\alpha)+\Delta_{f}^{\prime} \\
& \gamma_{11 \ldots 1}(f)=\gamma(f)+\nu+g\left(\gamma_{11}-\gamma_{1}\right) .
\end{aligned}
$$

By the RG $\varepsilon$ expansion, the exponent $\gamma_{\mathbf{s}}(f)$ was found to be [18]

$$
\gamma_{\mathbf{s}}(f)=1+\left(\gamma_{1}-1\right) f-\left(\gamma_{1,1}+\nu\right) \frac{f(f-1)}{2}+f(f-1)(f-2) B(f),
$$

where $B(f)=c \varepsilon^{2}+\mathcal{O}\left(\varepsilon^{3}\right)$ and $c$ is an unknown constant. Colby et al. [10] and Shida et al. [14] performed Monte Carlo simulations to evaluate $\gamma_{\mathrm{s}}(f)$ (see section 3.1).

These exponents take different numbers depending upon whether the surface is repulsive, attractive, or "marginal" with respect to the monomers forming the arms of the star [18]. Here we call a surface "marginal" if the system is right at 
the adsorption transition where for $l \rightarrow \infty$ the chain configuration changes from $d$ dimensional to $(d-1)$-dimensional due to the attractive monomer-wall interaction.

Here, we draw attention to other properties of star polymers such as distribution functions $[18,19]$. In the semi-infinite geometry, we also expect $R_{\mathrm{C}} \sim R_{\text {gyr }} \sim R_{\mathrm{CE}} \sim$ $l^{\nu}$ as $(25)(\nu=3 / 4$ in two dimensions and $\nu=0.588$ in three dimensions $)$. In the case of center-absorbed stars, a dependence on the distances parallel and perpendicular to the surface $\left(\mathbf{r}_{\|}, z\right)$ appears. The monomer density profile behaves as

$$
\rho\left(\mathbf{r}_{\|}, z\right)=L\left(R_{\mathrm{C}}\right)^{-d} \psi_{\mathrm{s}}\left(\frac{r_{\|}}{R_{\mathrm{C}}}, \frac{z}{R_{\mathrm{C}}}\right),
$$

with the short distance behaviour of the scaling function $\psi_{\mathrm{s}}(x, y)$

$$
\psi_{\mathrm{s}}(x, 0) \sim x^{-d+\lambda(f)}, \quad \psi_{\mathrm{s}}(0, y) \sim y^{-d+1 / \nu}
$$

Equation (35) contains a new exponent $\lambda(f)$ which depends on $f$. This $\lambda(f)$ should coincide with $1 / \nu$ in the limit $f \rightarrow \infty$ where the Daoud-Cotton theory [3] or the cone picture [23] is applicable. The value of $\lambda(f)$ was determined with the $\varepsilon$ expansion by Ohno and Binder [19] and is given by $(f-1) \varepsilon / 4+\mathcal{O}\left(\varepsilon^{2}\right)$; it was also evaluated by means of Monte Carlo simulations by Shida et al. [14] (see section 3.1).

The center-end distribution function $g_{\mathrm{s}}\left(\mathbf{r}_{\|}^{\mathrm{CE}}, z^{\mathrm{E}}\right)$ behaves as

$$
g_{\mathrm{s}}\left(\mathbf{r}_{\|}^{\mathrm{CE}}, z^{\mathrm{E}}\right)=\left(R_{\mathrm{CE}}\right)^{-d} \phi_{\mathrm{s}}\left(\frac{r_{\|}^{\mathrm{CE}}}{R_{\mathrm{CE}}}, \frac{z^{\mathrm{E}}}{R_{\mathrm{CE}}}\right),
$$

where the scaling function $\phi(x, y)$ has the following short distance behaviour:

$$
\begin{aligned}
& \phi_{\mathrm{s}}(x, 0) \sim x^{\theta_{\|}(f)}, \quad \phi_{\mathrm{s}}(0, y) \sim y^{\theta_{\perp}(f)} \\
& \theta_{\|}(f)=\left[\gamma_{1}-\gamma_{\mathrm{s}}(f+1)+\gamma_{\mathrm{s}}(f)-1\right] / \nu \\
& \theta_{\perp}(f)=\left[\gamma-\gamma_{\mathbf{s}}(f+1)+\gamma_{\mathbf{s}}(f)-1\right] / \nu
\end{aligned}
$$

Here the exponents $\gamma_{1},\left(\gamma_{11}\right)$ refer to the number of configuration of a linear chain polymer with one end, (two ends) grafted at a surface [54,55]. These relations were tested for $f=2-15$ stars by means of Monte Carlo simulations by Shida et al. [14].

Equations (34)-(39) hold not only for a repulsive wall but also for a "marginal wall", where the adsorption transition from $d$-dimensional to $(d-1)$-dimensional behaviours takes place. For a marginal wall, the exclusion effect against polymers by the hard wall is cancelled by a sufficient strength of the attractive wall interaction. For slightly stronger attractive walls, polymers are adsorbed on the wall and exhibit $(d-1)$-dimensional behaviour. In the polymer-magnetism analogy, this behaviour corresponds to the surface transition above the bulk $T_{\mathrm{c}}$ of a magnetic system where the coupling constant at the surface $\left(K_{\mathrm{s}}\right)$ exceeds that in the bulk $(K)$. The polymer problems near a marginal wall correspond in magnetic analogy to the critical behaviour at a "special transition" or a "surface-bulk" (SB) multicritical point [55]. The scaling relation (30) for networks with grafted chains also applies to the adsorption transition if $\Delta_{h}^{\prime}$ is replaced by $\Delta_{h}^{\mathrm{SB}}$. For example, $\Delta_{2}^{\mathrm{SB}}$ is no more given by 
$-\nu$ but by $\varphi$, which is the "crossover exponent" [54,55]. The $\gamma_{\mathcal{G}}$ obeys the scaling relation

$$
\gamma_{\mathcal{G}}^{\mathrm{SB}}=\alpha-1-f+\nu+\sum_{h=1}^{\infty}\left[n_{h} \Delta_{h}+n_{h}^{\prime} \Delta_{h}^{\mathrm{SB}}\right] .
$$

$\Delta_{h}$ and $\Delta_{h}^{\mathrm{SB}}$ are related, respectively, to the exponents of star polymers via (21) and

$$
\gamma_{\mathrm{s}}^{\mathrm{SB}}(f)=\alpha-1+\nu+\frac{f}{2}(\gamma-\alpha)+\Delta_{f}^{\mathrm{SB}} \text {. }
$$

Note that these equations (40),(41) have exactly the same form as the repulsive case [see equations $(30),(31)]$, if we replace $\Delta_{h}^{\mathrm{SB}}$ by $\Delta_{h}^{\prime}$. First several $\Delta^{\prime}$ 's are expressed with only the exponents of a linear polymer:

$$
\begin{aligned}
& \Delta_{1}=1+(\gamma-\alpha) / 2, \quad \Delta_{2}=1, \quad \Delta_{1}^{\prime}=(d-1) \nu / 2+\gamma_{11} / 2, \quad \Delta_{2}^{\prime}=-\nu, \\
& \Delta_{1}^{\mathrm{SB}}=(d-1) \nu / 2+\gamma_{11}^{\mathrm{SB}} / 2, \quad \Delta_{2}^{\mathrm{SB}}=\varphi .
\end{aligned}
$$

For the attractive surface, equations (34)-(39) need modification, since the characteristic lengths parallel and perpendicular to the surface differ and are given by

$$
\begin{array}{rlrl}
R_{\mathrm{CE}, \|} & \sim R_{\mathrm{C}, \|} \sim R_{\mathrm{gyr}, \|} \sim l^{\nu^{(d-1)}}, & & l \rightarrow \infty, \\
R_{\mathrm{CE}, \perp} \sim R_{\mathrm{C}, \perp} \sim R_{\mathrm{gyr}, \perp} \sim|c|^{-1} \sim \text { const }, & l \rightarrow \infty .
\end{array}
$$

Instead of equations (34) and (35), we have

$$
\rho\left(\mathbf{r}_{\|}, z\right)=L\left(R_{\mathrm{C}, \|}\right)^{-(d-1)}\left(R_{\mathrm{C}, \perp}\right)^{-1} \psi_{\mathrm{s}}\left(\frac{r_{\|}}{R_{\mathrm{C}, \|}}, \frac{z}{R_{\mathrm{C}, \perp}}\right)
$$

with $\psi_{\mathrm{s}}(x, 0) \sim x^{-(d-1)+1 / \nu^{(d-1)}}$. That is, the asymptotic properties of a star at an attractive wall are the same as those of a star in a $(d-1)$-dimensional geometry. Similar to (44), we have for the center-end distribution function

$$
g_{\mathrm{s}}\left(\mathbf{r}_{\|}^{\mathrm{CE}}, z^{\mathrm{E}}\right)=\left(R_{\mathrm{CE}, \|}\right)^{-(d-1)}\left(R_{\mathrm{CE}, \perp}\right)^{-1} \phi_{\mathrm{s}}\left(\frac{r_{\|}^{\mathrm{CE}}}{R_{\mathrm{CE}, \|}}, \frac{z^{\mathrm{E}}}{R_{\mathrm{CE}, \perp}}\right),
$$

with $\phi_{\mathrm{s}}(x, 0) \sim x^{\theta^{(d-1)}(f)}$, and $\theta^{(d-1)}(f)$ given by an equation similar to (29) but with all exponents taking their $(d-1)$-dimensional values. Note that equations (42)-(45) are valid only for $d>2$, since for $d=2$ only two arms of a star polymer would be adsorbed on the surface, and the configuration of the remaining arms would be just that of a star with $f-2$ arms at a repulsive wall.

At the adsorption transition, $\gamma_{\mathbf{s}}(f)$ and $\gamma_{1}$ in equations (34)-(39) must be replaced by $\gamma_{\mathrm{s}}^{\mathrm{SB}}(f)$ and $\gamma_{11}^{\mathrm{SB}}$. Using the RG $\varepsilon(=4-d)$ expansion, Ohno and Binder [18] found

$$
\gamma_{\mathrm{S}}^{\mathrm{SB}}(f)=1+\left(\gamma_{1}^{\mathrm{SB}}-1\right) f+\left(\varphi-\gamma_{11}^{\mathrm{SB}}\right) \frac{f(f-1)}{2}+f(f-1)(f-2) C(f) .
$$

In equation (46), $C(f)$ is a polynomial of both $\varepsilon$ and $f$ and of order $\varepsilon^{2}, \varphi$ is the crossover exponent, and $\gamma_{1}^{\mathrm{SB}},\left(\gamma_{11}^{\mathrm{SB}}\right)$ are the multicritical values of the conformation number exponents $\gamma_{1},\left(\gamma_{11}\right)$ of linear polymers with one, (two) ends at the surface. 


\section{Numerical simulations}

Using various computer simulation techniques, one can simulate star polymers in solvents. Using the molecular dynamics method, Grest et al. [8] have investigated gyration radius of star polymers with up to 50 arms. Grest et al. also investigated relaxation times of star polymers [43]. To investigate the total number of configurations, it is necessary to count the probability of elongating arms (with sufficiently long chains to get to the scaling regime). For this purpose, Monte Carlo techniques have been used successfully. Off-lattice Monte Carlo simulations have been performed by Rey et al. [45], Freire et al. [47] and Rubio and Freire [41]. Lattice Monte Carlo simulations of star polymers with up to 6 arms have been done by Lipson et al. [6] and Wilkinson et al. [7,46]. Batouris and Kremer [11] used a biased sampling method in their Monte Carlo simulations. Ohno and Binder [12,13] and Shida et al. [48] have applied an efficient enrichment algorithm to star polymers which enables one to treat star polymers with relatively large number of arms $(\sim 32)$. Colby et al. [10] and Shida et al. [14] investigated grafted star polymers in a semi-infinite geometry. There is also an application of static Monte Carlo techniques to the investigation of a relaxation time by Ohno et al. [44].

\subsection{Enrichment algorithm}

In the enrichment algorithm for star polymers [12-14,44,48], we generate monodisperse $f$-arm stars with arm length $l+1$ from those with arm length $l$ which is shorter by one segment, using a standard Monte Carlo technique. The number of arms, $f$, is fixed throughout the computation. On a simple cubic lattice, for example, we have five ways of elongating one end of an arm by one segment, because the 6th direction makes the arm fold backwards on itself. The success ratio is given by $(\mu / 5)^{f}=(0.93706)^{f}$ (the effective coordination number of the simple cubic lattice is $\mu=4.6853$ [62]). Then, one-step larger star polymers are generated by elongating every arm by one segment. At each step, the self-avoiding condition is tested; unless this condition is fulfilled, generated configurations are simply discarded.

If we had to consider all possible realizations at the $l+1$ st step from $M_{l}$ distinct realizations at the $l$ th step, we would have to make all $5^{f} M_{l}$ trials. However, doing only $m M_{l}$ trials $\left(m \ll 5^{f}\right)$ which are much less than the full $5^{f} M_{l}$ trials, we can collect a sufficient number of samples which are statistically isomorphic to the full realizations. That is, we generate only a limited number of samples $M_{l+1}$ from $M_{l}$ samples by a Monte Carlo method. This process can be iterated when $M_{l} \approx M_{l+1} \approx$ ... The condition $M_{l+1} / M_{l} \geqslant 1$ is satisfied when we choose $m \geqslant(5 / \mu)^{f}=(1.0672)^{f}$. In practice, it is necessary to increase $M_{l}$ gradually as $l$ increases, because one has to avoid an unphysical "bias" caused from this iteration. However, even for $f$ as large as $f=18$ a rather small value of $m(\approx 10)$ results. (For small $l$, it is better to work with somewhat larger values of $m$.) This enrichment algorithm for star polymers significantly reduces computing time, since it becomes quite efficient asymptotically for very long arms. In contrast, the conformation of shorter stars, typically with $l=1$ or 2 , are more rapidly counted by the direct SAW algorithm. 
Table 1. The values of the exponent $\gamma(f)$ for $f$-arm star polymers with $f=$ $3-6,12,18,32$ estimated by Monte Carlo simulations.

\begin{tabular}{ccccc}
\hline$f$ & 3 & 4 & 5 & 6 \\
\hline$[7]$ & $1.05 \pm 0.03$ & $0.88 \pm 0.03$ & $0.55 \pm 0.05$ & $0.20 \pm 0.05$ \\
\hline$[11]$ & $1.089 \pm 0.001$ & $0.879 \pm 0.001$ & $0.567 \pm 0.002$ & $0.16 \pm 0.01$ \\
\hline$f$ & 12 & 18 & 32 & 6 \\
\hline$[13]$ & $-3.4 \pm 0.3$ & $-8.9 \pm 0.2$ & $-29 \pm 2$ & $0.18 \pm 0.05$ \\
\hline
\end{tabular}

Table 2. The values of the exponents $\gamma(f)$ and $\lambda(f)$ for $f$-arm star polymers with $f=2-6,8,10,12,15$ estimated by lattice enrichment Monte Carlo simulations [14].

\begin{tabular}{cccccccccc}
\hline$f$ & 2 & 3 & 4 & 5 & 6 & 8 & 10 & 12 & 15 \\
\hline$\gamma_{\mathrm{s}}(f)$ & 0.10 & -0.52 & -1.21 & -2.03 & -2.92 & -4.9 & -7.1 & -9.4 & -13 \\
\hline$\lambda(f)$ & 0.8 & 0.9 & 0.9 & 1.0 & $1 . l$ & 1.1 & 1.3 & 1.4 & 1.5 \\
\hline
\end{tabular}

The total number of configurations of $f$-arm stars with each arm length equal to $l$ as its successive ratio $\mathcal{N}_{l+1} / \mathcal{N}_{l}$ can be identified as $5^{f} M_{l+1} / m M_{l}$. If we consider alternatively $\left(\mathcal{N}_{l+1} / \mathcal{N}_{l}\right)^{1 / f}$, we have, from $\mathcal{N}_{l} \sim \mu^{f l} l^{\gamma(f)-1}$,

$$
\left(\frac{\mathcal{N}_{l+1}}{\mathcal{N}_{l}}\right)^{\frac{1}{f}}=\left(\frac{5^{f} M_{l+1}}{m M_{l}}\right)^{\frac{1}{f}}=\mu\left\{1+\frac{\gamma(f)-1}{f l}\left[1+\frac{\gamma(f)-1-f}{2 f l}\right]+\mathcal{O}\left(\frac{1}{l^{3}}\right)\right\} .
$$

Since the quadratic correction becomes negligible for $l \gg|\gamma(f)-1-f| /(2 f)$, the configuration number exponent $\gamma(f)$ can be determined from a plot of $\left(5^{f} M_{l+1} / m M_{l}\right)^{1 / f}$ versus $1 / f l$ : if the numerical data fall on a straight line with the (known) intersection $\mu^{f}$ on the ordinate axis, the slope of this straight line yields $[\gamma(f)-1] \mu$. The enrichment algorithm was recently applied to the semi-dilute regime of linear chain solutions [63], although its extension to star polymer solutions has not been done yet.

Using the enrichment algorithm, Ohno [13] generated 6-, 12-, 18- and 32-arm star polymers with an octahedral core as center unit. The resulting exponent $\gamma(f)$ is listed in table 1 together with earlier works for up to 6-arm stars by Wilkinson et al. [7] and Batoulis and Kremer [11]. The exponents $\gamma_{\mathrm{s}}(f)$ and $\lambda(f)$ [see (35) for its definition] for center-adsorbed stars in a semi-infinite geometry were evaluated by Shida et al. [14] (first several numbers for $\gamma_{\mathrm{s}}(f)$ were also estimated by Colby et al. [10]). They are listed in table 2. As was pointed out by Ohno and Binder [18], $\lambda(f)$ is an increasing function of $f$ and approaches $1 / \nu \approx 1.70$ as $f$ increases.

The data also allow a significant study of linear dimensions such as the mean square distance from the center, $R_{\mathrm{C}}$, and the mean square distance $R_{\mathrm{CE}}$ between 
the end $(\mathrm{E})$ of an arm and the center $(\mathrm{C})$. All results $[6,7,11-13]$ are consistent in high accuracy with the Daoud-Cotton prediction (25) for the $f$ and $l$ dependences.

\subsection{Virial coefficient}

Entropic properties of star polymers in good solvents are closely related to the total number of configurations. Let us consider the osmotic pressure $\Pi$ of a solution of monodisperse star polymers, which have $f$ arms, each consisting of $l$ segments. In a dilute solution, $\Pi$ is expressed in a power series of the concentration $c$ as

$$
\frac{\Pi}{N_{\mathrm{A}} k_{\mathrm{B}} T}=\frac{c}{M}+A_{2} c^{2}+A_{3} c^{3}+\ldots
$$

where $N_{\mathrm{A}}$ stands for Avogadro's number, and $M=f l m$ means the molecular weight of one star polymer ( $m$ is the molecular weight of one segment). This series is usually called the virial expansion, and $A_{i}(i=2,3, \ldots)$ is referred to as the $i$ th virial coefficient of the solution. Available information about the virial coefficients is largely limited to $A_{2}$, because of the difficulty in estimating $A_{3}$ and higher coefficients.

Consider two star polymers in a solution, and write the $i$ th joint point between two adjacent segments of the first (second) star as $\boldsymbol{r}_{i}\left(\boldsymbol{\sigma}_{i}\right)$ and the center of the first (second) star as $\boldsymbol{r}_{0}\left(\boldsymbol{\sigma}_{0}\right)$. In the good solvent limit, the two body interaction $u\left(\boldsymbol{r}_{i}-\boldsymbol{\sigma}_{j}\right)$ is given by $(\alpha / \beta) \delta\left(\boldsymbol{r}_{i}-\boldsymbol{\sigma}_{j}\right)$ with $\beta=1 / k_{\mathrm{B}} T$ and $\alpha(>0)$ being the excluded-volume parameter. Then, the second virial coefficient $A_{2}$ can be expressed as

$$
A_{2}=-\frac{N_{\mathrm{A}}}{2 N M^{2}} \sum_{\{\boldsymbol{r}\}} \sum_{\{\boldsymbol{\sigma}\}} P\{\boldsymbol{r}\} P\{\boldsymbol{\sigma}\}\left\{\prod_{i=0}^{L} \prod_{j=0}^{L}\left[1-\delta \boldsymbol{r}_{i}, \boldsymbol{\sigma}_{j}\right]-1\right\},
$$

where $N$ is the volume of the solution (i.e., the number of lattice points), and $P\{\boldsymbol{r}\}$ is the one-body distribution function of a star polymer normalized as $\sum_{\{\boldsymbol{r}\}} P\{\boldsymbol{r}\}=N$ (the $\{\boldsymbol{r}\}$-sum is taken by $\boldsymbol{r}_{0}$ as well). The total number of configurations $\mathcal{N}(l, D)$ of two star polymers apart at a vector distance $\boldsymbol{D}=\boldsymbol{r}_{0}-\boldsymbol{\sigma}_{0}$ is expressed as

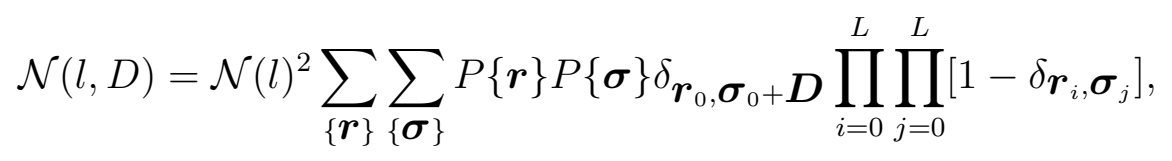

where $\mathcal{N}(l)$ denotes the total number of configurations of an isolated star polymer. Then, we find the following relation between $A_{2}$ and $\mathcal{N}(l, D)$ :

$$
\frac{A_{2} M^{2}}{N_{\mathrm{A}}}=-\frac{1}{2} \sum_{\boldsymbol{D}}\{g(l, D)-1\}, \quad g(l, D)=\frac{\mathcal{N}(l, D)}{\mathcal{N}(l, \infty)}
$$

Here we used $\mathcal{N}(l)^{2}=\mathcal{N}(l, \infty)$ which guarantees no interference at $D=\infty$. The function $g(l, D)$ in equation (51) is the pair distribution function in the dilute limit. It approaches zero for small $D$ as $D^{a_{2}}$ (with $a_{2}>0$ ) and goes to unity for large $D$. It is 
related to the effective interstar potential $U(l, D)$ via $g(l, D) \propto \exp \left[-U(l, D) / k_{\mathrm{B}} T\right]$. Then, we obtain the logarithmic dependence of the effective interstar potential

$$
U(l, D) \sim-a_{2} k_{\mathrm{B}} T \log D,
$$

which was first predicted by Witten and Pincus [29].

The penetration function $\Psi$, which is a combination of the second virial coefficient and the mean square radius of gyration as

$$
\Psi=\frac{A_{2} M^{2}}{\left.4 \pi^{3 / 2} N_{\mathrm{A}} R_{\mathrm{gyr}}^{2}\right)^{3 / 2}},
$$

is often used instead of $A_{2}$ itself, since it is known to be a universal quantity [37].

Ohno et al. [40] and Shida et al. [42] performed Monte Carlo simulations based on the enrichment algorithm to obtain the effective interstar potential and the second virial coefficient of star polymers. Rubio and Freire [41] evaluated the second virial coefficient of star polymers by using off-lattice Monte Carlo simulations.

In the enrichment algorithm, the successive ratios $\mathcal{N}(l, D) / \mathcal{N}(l-1, D)$ of the total number of configurations are obtained automatically. Multiplying these ratios from $l(\gg 10)$ to $l=10$ yields $N(l, D) / N(9, D)$. Then, $g(l, D)$ in $(51)$ is given by

$$
g(l, D)=\frac{N(l, D)}{N(9, D)} \frac{N(9, \infty)}{N(l, \infty)}=\frac{\frac{N(l, D)}{N(l-1, D)} \frac{N(l-1, D)}{N(l-2, D)} \frac{N(l-2, D)}{N(l-3, D)} \cdots \frac{N(11, D)}{N(10, D)} \frac{N(10, D)}{N(9, D)}}{\frac{N(l, \infty)}{N(l-1, \infty)} \frac{N(l-1, \infty)}{N(l-2, \infty)} \frac{N(l-2, \infty)}{N(l-3, \infty)} \cdots \frac{N(11, \infty)}{N(10, \infty)} \frac{N(10, \infty)}{N(9, \infty)}}
$$

In the second equality, we used $\mathcal{N}(9, D)=N(9, \infty)=N^{2}(9)$ for sufficiently large $D$. (In fact this relation holds for all $D$ satisfying $D>18$.) Then the substitution of equation (54) into equation (51) yields $A_{2}$ which can be evaluated from the subsequent ratios of the total number of configurations. The values for the gyration radius $R_{\text {gyr }}$, the second virial coefficient $A_{2}$, the penetration function $\Psi$ and the coefficient of the interstar potential $a_{2}$ obtained by Ohno et al. [40] are summarized in table 3. The results were successfully compared with the RG $\varepsilon$ expansion by Douglas and Freed [22], the prediction by Witten and Pincus [29] and the experiments for $\Psi$ by Douglas et al. [37], Roovers et al. [49] and Okumoto et al. [50].

Table 3. The estimated values [40] of the mean-square radius of gyration $R_{\text {gyr }}^{2}$, the second virial coefficient $A_{2} M^{2} / N_{\mathrm{A}}$, the penetration function $\Psi=$ $\left(A_{2} M^{2}\right) /\left(4 \pi^{3 / 2} N_{A} R_{\text {gyr }}^{3}\right)$ and the coefficient of the repulsive potential $a_{2}$.

\begin{tabular}{ccccc}
\hline$f$ & 3 & 4 & 5 & 6 \\
\hline$R_{\text {gyr }}^{2}$ & 91.38 & 103.08 & 111.14 & 118.11 \\
\hline$A_{2} M^{2} / N_{\mathrm{A}}$ & 6735.78 & 10622.13 & 13760.61 & 18534.96 \\
\hline$\Psi$ & 0.35 & 0.46 & 0.53 & 0.64 \\
\hline$a_{2}$ & 2.04 & 2.43 & 3.28 & 3.50 \\
\hline
\end{tabular}




\subsection{Relaxation time}

In the presence of excluded volume interaction, each arm is approximately restricted in its configuration to a cone the angle of which vanishes proportional to $1 / r^{1 / 2}$ as $f \rightarrow \infty$. For this situation, Grest et al. [43] suggested the existence of several relaxation times; the autocorrelation time $\tau_{\mathrm{B}}$ of the distance between a core and an arm end is associated with the size of the largest blob: $\xi_{\max } \propto R(f)^{-1 / 2}$, which contains $l_{\mathrm{Bmax}} \propto \xi_{\max }^{1 / \nu} \propto l f^{-1 / 2 \nu}$ effective monomers. Then Grest et al. [43] proposed

$$
\tau_{B} \propto \xi_{\max }^{2} l_{\mathrm{Bmax}} \propto l^{1+2 \nu} f^{-(1+2 \nu) / 2}, f \gg 1 .
$$

For a relaxation time for the shape fluctuation of a star polymer, where a density fluctuation has to diffuse a distance of the radius $R$ of the star polymer, they proposed a formula ("el" stands for "elastic")

$$
\tau_{\mathrm{el}} \propto \tau_{\mathrm{B}}\left(R / \xi_{\max }\right)^{2} \propto l^{1+2 \nu} f^{1-[(1+2 \nu) / 2]} \approx l^{2.176} f^{-0.088} .
$$

Grest et al. [43] performed molecular dynamics simulations of multi-arm star polymers and confirmed these relations as a function of $f$. Equation (56) was also confirmed by Ohno et al. [44] who used the enrichment Monte Carlo simulation combined with the Kramers potential method.

\subsection{Hydrodynamic interactions}

In the calculation of hydrodynamic properties, one often uses a rigid-body approximation, in which polymer chains in the flow are assumed to move as if they were rigid molecules, i.e., their equilibrium conformations are presurved in the flow. This leads to the Kirkwood-Riseman equation [64] for each polymer molecule,

$$
\frac{1}{6 \pi \eta_{0} a} \boldsymbol{F}_{i}+\sum_{i \neq j} \boldsymbol{T}_{i j} \boldsymbol{F}_{j}-\boldsymbol{u}_{i}=-\boldsymbol{v}_{i}^{0}
$$

Here, $\eta_{0}$ is the viscosity of the fluid, $a$ is the hydrodynamic radius of each segment, $\boldsymbol{F}_{i}=\left(F_{i x}, F_{i y}, F_{i z}\right)$ the frictional force exerted on the $i$ th segment, and $\boldsymbol{T}_{i j}$ is the Oseen tensor defined by

$$
\boldsymbol{T}_{i j}=\frac{1}{8 \pi \eta_{0} r_{i j}}\left\{\boldsymbol{I}+\frac{\boldsymbol{r}_{i j} \boldsymbol{r}_{i j}}{r_{i j}^{2}}\right\} .
$$

Here $\boldsymbol{r}_{i j}$ is the distance between the $i$ th and $j$ th segments.

From these equations, the hydrodynamic radius $R_{\mathrm{H}}$ can be calculated by setting the angular velocity at zero, and the intrinsic viscosity $\eta$ can be calculated by setting both the total force and total moment to be equal to zero. Such a treatment with Monte Carlo simulations was first given for a linear chain in 1956 by Zimm [65] and therefore is nowadays called a Zimm model. The Monte Carlo investigation for star polymers was first performed in 1986-1987 by Rey et al. [45] and then in 1988 
by Wilkinson et al. [46], and recently by Shida et al. [48]. Table 4 lists the results by Shida et al. [48] of the factors for the intrinsic viscosity and the hydrodynamic radius,

$$
\eta=B(f) L^{\nu}, \quad R_{\mathrm{H}}=C(f) L^{\nu}
$$

as well as the Flory viscosity factor

$$
\Phi \equiv[\eta] M /\left(6 R_{\text {gyr }}^{2}\right)^{3 / 2}
$$

and the hydrodynamic factor

$$
\rho \equiv R_{\mathrm{gyr}} / R_{\mathrm{H}}
$$

The data of $10^{-23} \Phi \mathrm{mol}^{-1}$ for $f=2$ and 12 obtained by Shida et al. [48] are close to those (1.80 and 5.18 for $f=2$ and $f=12$, respectively) obtained by Freire et al. [47] who used simulations without the rigid-body approximation. This implies that the approximation does not make much of an error on viscosity values. Table 4 also contains the values obtained from the empirical formulae for $g_{\eta} \equiv B(f) / B(2)$ and $g_{\mathrm{H}} \equiv C(f) / C(2)$, which were proposed by Douglas et al. [37] by fitting to their experimental data. As is seen in this table, the agreement between the Monte Carlo results and the empirical formulae is excellent.

Table 4. Monte Carlo results [48] of the factors for the intrinsic viscosity, $B(f)$ and $g_{\eta} \equiv B(f) / B(2)$, and for the hydrodynamic radius, $C(f)$ and $g_{\mathrm{H}} \equiv C(f) / C(2)$. The values obtained from empirical formulae by Douglas et al. [37] are also listed in the columns indicated by "Emp.". Results [48] of the Flory viscosity factor $\Phi$ and the hydrodynamic factor $\rho$ defined, respectively, by (60) and (61) are compared with experimental values [31-37] (the columns indicated by "Exp.").

\begin{tabular}{cccccccc}
\hline$f$ & 2 & 3 & 4 & 6 & 8 & 12 & 18 \\
\hline$B(f)$ & 1.10 & 0.95 & 0.80 & 0.61 & 0.49 & 0.37 & - \\
\hline$g_{\eta}$ & - & 0.86 & 0.73 & 0.55 & 0.45 & 0.34 & 0.25 \\
\hline Emp. $([37])$ & - & 0.83 & 0.71 & 0.56 & 0.46 & 0.33 & 0.22 \\
\hline$C(f)$ & 0.41 & 0.38 & 0.37 & 0.35 & 0.33 & 0.30 & 0.27 \\
\hline$g_{\mathrm{H}}$ & - & 0.94 & 0.92 & 0.86 & 0.82 & 0.73 & 0.66 \\
\hline Emp. $([37])$ & - & 0.96 & 0.92 & 0.86 & 0.80 & 0.72 & 0.63 \\
\hline $10^{-23} \Phi \mathrm{mol}^{-1}$ & 2.0 & 2.3 & 2.9 & 3.7 & 4.3 & 5.8 & 7.2 \\
\hline Exp. $([31-37])$ & - & 2.6 & 3.1 & $3.3-3.9$ & 4.1 & $5.5-6.1$ & 5.8 \\
\hline$\rho$ & 1.36 & 1.26 & 1.16 & 1.04 & 0.97 & 0.88 & 0.80 \\
\hline Exp. $([31-37])$ & - & - & 1.09 & 1.01 & - & 0.92 & 0.88 \\
\hline
\end{tabular}




\section{References}

1. Zimm B.H., Stockmayer W.H. The dimensions of chain molecules containing branches and rings. // J. Chem. Phys., 1949, vol. 17, No. 12, p. 1301-1314.

2. de Gennes P.G. Scaling Concepts in Polymer Physics. Ithaca, Cornell University Press, 1979.

3. Daoud M., Cotton J.P. Star shaped polymers: a model for the conformation and its concentration dependence // J. Phys. (France), 1982, vol. 43, No. 3, p. 531-538.

4. Binder K. Introduction: general aspects of computer simulation techniques and their applications in polymer physics. - In: Monte Carlo and Molecular Dynamics Simulations in Polymer Science. Binder, K. (Ed.), Oxford, Oxford University Press, 1995, p. $476-578$.

5. Grest G.S., Murat M. Computer simulations of tethered chains. - In: Monte Carlo and Molecular Dynamics Simulations in Polymer Science. Binder, K. (Ed.), Oxford, Oxford University Press, 1995, p. 1-46.

6. Lipson J.E.G., Whittington S.G., Wilkinson M.K., Martin J.L., Gaunt D.S. A lattice model of uniform star polymers. // J. Phys. A: Math. Gen., 1985, vol. 18, No. 8, p. L469-L473.

7. Wilkinson M.K., Gaunt D.S., Lipson J.E.G., Whittington S.G. Lattice models of branched polymers: statistics of uniform stars. // J. Phys. A: Math. Gen., 1986, vol. 19, No. 5, p. $789-796$.

8. Grest G.S., Kremer K., Witten T.A. Structure of many-arm star polymers: a molecular dynamics simulation. // Macromolecules, 1987, vol. 20, No. 6, p. 1376-1383.

9. Barrett A.J., Tremain D.L. Lattice walk models of uniform star polymers with many arms. // Macromolecules, 1987, vol. 20, No. 7, p. 1687-1692.

10. Colby S.A., Gaunt D.S., Torrie G.M., Whittington S.G. Branched polymers attached in a wedge geometry. // J. Phys. A: Math. Gen., 1987, vol. 20, No. 8, p. L515-L520.

11. Batoulis, J., Kremer, K. Thermodynamic properties of star polymers: good solvents. // Macromolecules, 1989, vol. 22, No. 11, p. 4277-4285.

12. Ohno K., Binder K. Monte Carlo simulation of manay-arm star polymers in twodimensional good solvents in the bulk and at a surface. // J. Stat. Phys., 1991, vol. 64, No. 3-4, p. 781-806.

13. Ohno K. Monte Carlo simulation of many-arm star polymers. // Macromolecular Symposia, 1994, vol. 81, No. 1, p. 121-127.

14. Shida K., Ohno K., Kimura M., Kawazoe Y. Large scale Monte Carlo simulations of center-adsorbed star polymers. // J. Chem. Phys., 1996, vol. 105, No. 19, p. 8929-8936.

15. Duplantier B. Polymer network of fixed topology: renormalization, exact critical exponent $\gamma$ in two dimensions, and $d=4-\varepsilon$. // Phys. Rev. Lett., 1986, vol. 57, No. 8, p. 941-944; Errata: ibid, 1986, vol. 57, No. 18, p. 2332.

16. Duplantier B., Saleur H. Exact surface and wedge exponents for polymers in two dimensions. // Phys. Rev. Lett., 1986, vol. 57, No. 25, p. 3179-3182.

17. Ohno K., Binder K. Scaling theory of star polymers and general polymer networks in bulk and semi-infinite solvents. // J. Phys. (France), 1988, vol. 49, No. 8, p. 1329-1351.

18. Ohno K., Binder K. Scaling theory for radial distributions of star polymers in dilute solution in the bulk and at a surface, and scaling of polymer networks near the adsorption transition. // J. Chem. Phys., 1991, vol. 95, No. 7, p. 5444-5458.

19. Ohno K., Binder K. Scaling theory for radial distributions of star polymers in dilute 
solution in the bulk and at a surface II. $\varepsilon$ expansion for monomer densities. // J. Chem. Phys., 1991, vol. 95, No. 7, p. 5459-5473.

20. Miyake A., Freed K.F. Excluded volume in star polymers: chain conformation space renormalization group. // Macromolecules, 1983, vol. 16, No. 7, p. 1228-1241.

21. Miyake A., Freed K.F. Internal chain conformations of star polymers. // Macromolecules, 1984, vol. 17, No. 4, p. 678-683.

22. Douglas J.F., Freed K.F. Penetration function and second virial coefficient for linear and regular star polymers. // Macromolecules, 1984, vol. 17, No. 9, p. 1854-1870.

23. Ohno K. Statistics of many-arm star polymers. // Phys. Rev. A, 1989, vol. 40, No. 3, p. $1524-1538$.

24. Duplantier B. Statistical methods for polymers and membranes: renormalization, conformal invariance and matrix models. - In: Polymer Physics, 25 Years of the Edwards Model. Bhattacharjee, S.M. (Ed.), Singapore, World Scientific, 1992, p. 7-81.

25. Schäfer L., von Ferber C., Lehr U., Duplantier B. Renormalization of polymer networks and stars. // Nucl. Phys. B, 1992, vol. 374, No. 3, p. 473-495.

26. Vlahos C.H., Kosmas M.K. Conformational properties of regular comb polymers. // J. Phys. A: Math. Gen., 1987, vol. 20, No. 6, p. 1471-1483.

27. Leibler L., Orland H., Wheeler J.C. Theory of critical micelle concentration for solutions of block copolymers. // J. Chem. Phys., 1983, vol. 79, No. 7, p. 3550-3557.

28. Higgins J.S., Dawkins J.V., Maghami G.G., Shakir S.A. Study of micelle formation by the diblock copolymer polystyrene-b-(ethylene-co-propylene) in dodecane by smallangle neutron scattering. // Polymer, 1986, vol. 27, p. 931-936.

29. Witten T.A., Pincus P.A. Colloid stabilization by long grafted polymers. // Macromolecules, 1986, vol. 19, No. 10, p. 2509-2513.

30. Birshtein T.M., Zhulina E.B. Scaling theory of supermolecular structures in block copolymer-solvent systems: 1. Model of micellar structures. // Polymer, 1989, vol. 30, p. $170-177$.

31. Meunier J.-C., Leemput R. Etude en solution diluée des propriétés du polystyrène ramifié en étoile. // Makromol. Chem., 1971, vol. 147, p. 191-211 (in French).

32. Roovers J.E.L, Bywater S. Preparation and characterization of four-branched star polystyrene. // Macromolecules, 1972, vol. 5, No. 4, p. 384-388.

33. Roovers J., Toporowski P.M. Hydrodynamic studies on model branched polystyrenes. // J. Polym. Sci.: Polym. Phys. Ed., 1980, vol. 18, p. 1907-1917.

34. Roovers J., Hadjichristidis N., Fetters L.J. Analysis and dilute solution properties of 12- and 18-arm star polystyrenes. // Macromolecules, 1983, vol. 16, No. 2, p. 214-220.

35. Khasat N., Pennisi R.W., Hadjichristidis N., Fetters L.J. Dilute solution behaviour of asymmetric three-arm and regular three- and twelve-arm polystyrene stars. // Macromolecules, 1988, vol. 21, No. 4, p. 1100-1106.

36. Bauer B.J., Fetters L.J., Graessley W.W., Hadjichristidis N., Quack, G.F. Chain dimsntions in dilute polymer solutions: a light scattering and viscometric study of multiarmed polyisoprene stars in good and $\theta$ solvents. // Macromolecules, 1989, vol. 22, No. 5, p. 2337-2347.

37. Douglas J.F., Roovers J., Freed K.F. Characterization of branching architecture through "universal" ratios of polymer solution properties. // Macromolecules, 1990, vol. 23, No. 18, p. 4168-4180.

38. Merkle G., Burchard W., Lutz P., Freed K.F., Gao J. Osmotic pressure of linear, star, and ring polymers in semidilute solution. A comparison between experiment and 
theory. // Macromolecules, 1993, vol. 26, No. 11, p. 2736-2742.

39. Roovers J. Concentration dependence of the relative viscosity of star polymers. // Macromolecules, 1994, vol. 27, No. 19, p. 5259-5364.

40. Ohno K., Shida K., Kimura M., Kawazoe Y. Monte Carlo study on second virial coefficient of star polymers in good solvent. // Macromolecules, 1996, vol. 29, No. 6, p. 2269-2274.

41. Rubio A., Freire J.J. Monte Carlo calculation of second virial coefficient for linear and star chains in a good solvent. // Macromolecules, 1996, vol. 29, No. 21, p. 6946-6951.

42. Shida K., Ohno K., Kimura M., Kawazoe Y. Monte Carlo study of the second virial coefficient and statistical exponent of star polymers with large numbers of branches. // Macromolecules, 2000, vol. 33. No. 20, p. 7655-7662.

43. Grest G.S., Kremer K., Milner S.T., Witten T.A. Relaxation of self-entangled manyarm star polymers. // Macromolecules, 1989, vol. 22, No. 4, p. 1904-1910.

44. Ohno K., Schulz M., Binder K., Frisch H.L. Dynamics of star polymers in a good solvent: a Kramers potential treatment. // J. Chem. Phys., 1994, vol. 101, No. 5, p. $4452-4460$.

45. Rey A., Freire J.J., de la Torre J.G. Monte Carlo calculations for linear and star polymers with intermolecular interactions. 3. Dimensions and hydrodynamic properties in good solvent conditions. // Macromolecules, 1987, vol. 20, No. 2, p. 342-346.

46. Wilkinson M.K., Gaunt D.S., Lipson J.E.G., Whittington S.G. Lattice models of branched polymers: dynamics of uniform stars. // Macromolecules, 1988, vol. 21, No. 6, p. $1818-1822$.

47. Freire J.J., Rey A., Bishop M., Clarke J.H.R. Dimensions and intrinsic viscosities of long linear and star chains in good and $\theta$ solvent conditions. // Macromolecules, 1991, vol. 24, No. 24, p. 6494-6499.

48. Shida K., Ohno K., Kimura M., Kawazoe Y. Dimensional and hydrodynamic factors for flexible star polymers in the good solvent limit. // Macromolecules, 1998, vol. 31, No. 7 , p. 2343-2348.

49. Roovers J., Toporowski P.M., Douglas J. Thermodynamic properties of dilute and semidilute solutions of regular star polymers. // Macromolecules, 1995, vol. 28, No. 21, p. $7064-7070$.

50. Okumoto M., Nakamura Y., Norisue T., Teramoto A. Excluded-volume effects in star polymer solutions: four-arm star polystyrene in benzene. // Macromolecules, 1998, vol. 31 , No. 5, p. 1615-1620.

51. Freire J.J. Conformational properties of branched polymers: theory and simulations. In: Advances in Polymer Science, 1999, vol. 143, Berlin, Heidelberg, Springer-Verlag, p. $35-112$.

52. de Gennes P.G. Some conformation problems for long macromolecules. // Rep. Prog. Phys., 1969, vol. 32, p. 187-205.

53. Binder K., Kremer K. Scaling Phenomena in Disordered Systems. R. Pynn and A. Skjeltorp (Ed.) New-York, Plenum Press, 1985.

54. Eisenriegler E., Kremer K., Binder K. Adsorption of polymer chains at surfaces: scaling and Monte Carlo analyses. // J. Chem. Phys., 1982, vol. 77, No. 12, p. 6296-6320.

55. Binder K. Critical behaviour at surfaces. - In: Phase Transitions and Critical Phenomena. Vol. 8, C. Domb and J. L. Lebowitz (Ed.), London, Academic Press, 1983, p. $1-144,467-474$.

56. Murat M., Grest G.S. Structure of a grafted polymer brush: a molecular dynamics 
simulation. // Macromolecules, 1989, vol. 22, No. 10, p. 4054-4059.

57. des Cloizeaux J. Polymers in solutions: principles and applications of a direct renormalization method. // J. Phys. (France), 1981, vol. 42, No. 5, p. 635-652.

58. Breézin E., Le Guillou J.-C., Zinn-Justin J. Field theoretical approach to critical phenomena. - In: Phase Transitions and Critical Phenomena. Domb C., Green M.S. (Ed.), 1974, Vol. 6, p. 125-247.

59. Duplantier B., Saleur H. Exact critical properties of two-dimensional dense selfavoiding walks. // Nucl. Phys. B, 1987, [FS20], vol. 290, No. 3, p. 291-326.

60. des Cloizeaux J. Stort range correlation between elements of a long polymer in good solvent. // J. Phys. (France), 1980, vol. 41, No. 3, p. 223-238.

61. Ohno K. Statistical mechanics of polymer networks. - In: Computationa Approaches in Condensed-Matter Physics. Miyashita S., Imada M., Takayama H. (Ed.), Springer Proceedings in Physics, 1992, Vol. 70, p. 276-277.

62. Watts M.G. Application of the method of Padé approximants to the excluded volume problem. // J. Phys. A: Math. Gen., 1975, vol. 8, p. 61-66.

63. Shida K., Ohno K., Kimura M., Kawazoe Y. Monte Carlo analysis of the osmotic pressure of athermal polymer solutions in dilute and semi-dilute regimes. // Comput. Theor. Polymer Sce., 2000, vol. 10, No. 3-4, p. 281-285.

64. Kirkwood J.G., Riseman J. The intrinsic viscosities and diffusion constants of flexible macromolecules in solution // J. Chem. Phys., 1948, vol. 16, No. 6, p. 565-573.

65. Zimm B.H. Dynamics of polymer molecules in dilute solution: viscoelasticity, flow birefringence and dielectric loss. // J. Chem. Phys., 1956, vol. 24, No. 2, p. 269-278. 


\section{Теорія скейлінгу і комп'ютерне моделювання зіркових полімерів в добрих розчинниках}

\section{К.Оно}

Кафедра фізики, технологічний факультет, Національний університет м. Йокогама, 79-5 Токівадаі, Годогая-ку, Йокогама 240-8501, Японія

Отримано 7 грудня 2001 р.

Проведено огляд теорій скейлінгу і ренормгрупового $\varepsilon=4-d$ розкладу ( $d$ - вимірність простору) а також комп'ютерного моделювання зіркових полімерів (і полімерних сіток), що складаються з довгих гнучких ланцюгів однакової довжини і знаходяться в розведеному розчині в границі доброго розчинника. Зокрема, в термінах аналогії полімер-магнетик детально обговорюється асимптотична поведінка конформаційних і ентропійних величин в границі довгих ланцюгів. Розглянуто не лише статичні характеристики, такі, як функції розподілу і осмотичний тиск чи ентропія, але і динамічні властивості, такі як час релаксації і власна в'язкість зіркових полімерів.

Ключові слова: ренормалізаційна група, моделювання методом Монте Карло, загальна кількість конфігурацій, віріальний коефіцієнт, час релаксації, гідродинамічний ефект

PACS: 05.10.Cc, 05.10.Ln, 61.41.+e, 82.35.Gh, 82.35.Lr, 82.70.Uv 